\title{
Efectos de un programa de Aprendizaje- Servicio en el alumnado universitario
}

\section{Mirian Hervás Torres ${ }^{1}$, Francisco D. Fernández Martín ${ }^{1}$, José Luis Arco Tirado ${ }^{1}$, María Isabel Miñaca Laprida ${ }^{2}$}

${ }^{1}$ Departamento de Psicología Evolutiva y de la Educación, Universidad de Granada, Granada

${ }^{2}$ Psicóloga, Asociación Nuevo Futuro, Madrid

\section{España}

Correspondencia: Mirian Hevás Torres. Departamento de Psicología Evolutiva y de la Educación, Universidad de Granada, Campus Universitario de Cartuja s/n, 18071, Granada (España)

e-mail: mirian.hervastorres@gmail.com

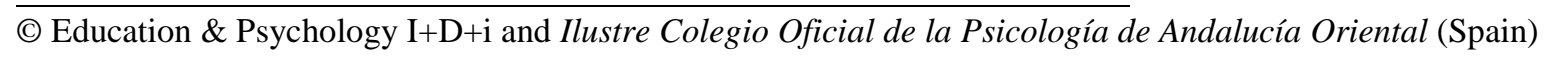




\section{Resumen}

Introducción. La baja productividad de la Educación Superior requiere urgentemente respuestas institucionales dirigidas a la mejora calidad de la enseñanza universitaria, que contribuyan al desarrollo entre el alumnado de competencias clave para el aprendizaje permanente. En este sentido, el propósito de esta investigación fue explorar los efectos de un programa de intervención, basado en las metodologías aprendizaje-servicio y mentoría entre iguales, dirigido a mejorar las competencias sociales y de aprender a aprender del alumnado universitario.

Método. La muestra estuvo compuesta por 78 alumnos/as universitarios/as de 5 titulaciones relacionadas con la profesión docente de la Universidad de Huelva (España). El diseño metodológico adoptado fue un diseño cuasi-experimental pretest-postest, empleando pruebas estandarizadas para evaluar las variables dependientes. La intervención consistió en 481 sesiones de mentoría, 423 individuales y 58 grupales, entre el alumnado universitario participante, en calidad de mentores, previamente entrenados en 3 sesiones de formación, y alumnado de educación obligatoria. Estas sesiones se extendieron a lo largo del segundo y tercer trimestre del curso escolar 2013/2014 en tiempo extraescolar, con una frecuencia de una sesión de mentoría semanal entre cada pareja de mentores y su correspondiente alumno/a, con una duración de 90 minutos.

Resultados. Los resultados obtenidos arrojan diferencias estadísticamente significativas, a favor de la fase postest en habilidades sociales y de las estrategias de aprendizaje y motivación de los participantes.

Discusión y conclusión. Estos resultados coinciden con las evidencias empíricas que la literatura especializada arroja cuando se emplean las metodologías de aprendizaje-servicio y mentoría entre iguales como base de un programa de intervención.

Palabras Clave: motivación, estrategias de aprendizaje, habilidades sociales, competencia, aprendizaje servicio. 


\begin{abstract}
Introduction. The low productivity that prevailing in recent years in Higher Education requires urgently institutional responses aimed to improving quality of university education contributing to the development among students of key competences for lifelong learning. In this sense, the aim of this research was to explore the effects of an intervention program, based on service-learning and peer mentoring methodologies, to enhance social skills and the learn to learn skill of university students.

Method. The sample was composed of 78 university students of 5 degrees related to the teaching profession of the University of Huelva (Spain). The methodological design adopted was quasi-experimental pretest-posttest, using standardized tests to evaluate the dependent variables. The intervention consisted of 481 mentoring sessions, 423 individual and 58 in group, among the university students, as mentors, previously trained in 3 sessions of training, and students in Compulsory Education. These sessions spread throughout the second and third quarters of the school year 2013/2014 during out of school time, a weekly mentoring session among the couple of mentors with their mentee lasted for 90 minutes each.
\end{abstract}

Results. The results show statistically significant differences in favour of the posttest phase in social skills and strategies for learning and motivation of the participants.

Discussion and Conclusion. These results agree largely whit the empirical evidences from specialized literature show when service-learning and peer mentoring methodology are used as the basis for an intervention program.

Keywords: motivation, learning strategies, social skills, competency, service learning. 


\section{Introducción}

En los últimos años Europa ha estado inmersa en una crisis que ha dejando a la luz las debilidades del sistema económico, político, educativo y social, tal y como reflejan los datos de la Unión Europea-27 respecto al gran riesgo de exclusión social, abandono de los estudios, alto número de desempleados y jóvenes que llegan al mercado laboral sin la cualificación suficiente (European Commission, 2013; Instituto Nacional de Estadística, 2015; Ministerio de Educación, Cultura y Deporte, 2015). Un claro indicador de esta situación es el elevado número de jóvenes que no está prosperando en su transición formativo-educativa al empleo de calidad y a su integración social, debido a barreras como la calidad en el sistema de formación y entrenamiento (Jurado, Olmos, y Pérez, 2014).

En este sentido, la baja productividad de la enseñanza universitaria, tanto en rendimiento académico inmediato (p.e., tasa de éxito del 86,7\%, tasa de rendimiento del 76,3\%, tasa de abandono del 26,9\% y tasa de graduación del 83,2\%; sólo el 10\% de la población con estudios superiores logra alcanzar niveles de rendimiento adecuados según el Programa Internacional para la Evaluación de las Competencias de la Población Adulta) (Ministerio de Educación, Cultura y Deporte, 2014, 2015) como diferido (p.e., el 55,5\% de los egresados 4 años después ocupan un puesto acorde a su titulación; opiniones negativas de los titulados respecto a las posibilidades que su formación ofrece para encontrar empleo y de los empresarios sobre la formación de los titulados; etc.) (Consejo Económico y Social de España, 2014; Freire, Teijeiro, y País, 2013; Ministerio de Educación, Cultura y Deporte, 2015), realzan claramente la necesidad de seguir avanzando en las reformas universitarias con actuaciones eficaces y eficientes que permitan mejorar eventualmente estos y otros indicadores de calidad y excelencia.

El mismo Consejo Económico y Social de España (2014), o autores como Mourshed, Patel, y Suder (2014), recomiendan a la enseñanza universitaria que incorpore metodologías educativas innovadoras que estimulen la mejora de las competencias clave, para el desarrollo personal del alumnado, participación activa en la sociedad, y tener éxito en el mundo laboral, potenciando aquellas habilidades demandadas desde el mercado de trabajo (p.e., experiencia práctica, ética en el trabajo, trabajo en equipo, análisis y resolución de problemas, creatividad, habilidades de comunicación e interacción social, autorregulación en el aprendizaje, etc.). 
En este contexto surge el Programa Huelva Educa (PHE), una experiencia educativa innovadora dirigida a mejorar el desarrollo de competencias y habilidades del alumnado universitario, especialmente las habilidades sociales y de aprender a aprender. En esta línea, las competencias sociales se conciben como aquellas habilidades específicas empleadas en una situación interpersonal determinada, incluyendo aquellas formas de comportamiento que preparan a los individuos para una participación eficaz en la vida social y profesional (Comunidades Europeas, 2007; Gismero, 2000). Por su parte, la competencia aprender a aprender, en los términos que se define como una competencia clave para el aprendizaje permanente (Comunidades Europeas, 2007), hace referencia al aprendizaje experto y la autorregulación en el aprendizaje, es decir, la participación activa en el aprendizaje en términos metacognitivos, motivacionales y comportamentales, donde las estrategias de aprendizaje y la motivación desempeñan un papel fundamental (Zimmerman, 2002).

Para conseguir este doble objetivo, el PHE incorpora en su diseño de intervención dos metodologías instruccionales capaces de transmitir estos aprendizajes al alumnado. Por un lado, la metodología alternativa denominada Aprendizaje-Servicio (A-S) (Westover, 2012; Wilczenski y Coomey, 2007), entendida como una metodología donde los participantes desarrollan habilidades, destrezas, competencias, etc., realizando un servicio a su comunidad. Y, por otro lado, el aprendizaje entre iguales, en su versión de mentoría (Herrera, Sipe, y McClanahan, 2000; Sandford, Copps, y Yeowart, 2007), donde una persona con experiencia ayuda a otra más joven en aspectos esenciales de su vida.

Entre los beneficios de este tipo de metodologías instruccionales destacan: (a) un aumento del compromiso del alumnado con el aprendizaje (Maloney y Griffith, 2013; McIntyre y Sellnow, 2014), (b) una mejora en la adquisición de los contenidos por parte del alumnado (McIntyre y Sellnow, 2014; Vaknin y Bresciani, 2013), (c) el fomento de ciertos aspectos relacionados con el desarrollo personal y social del alumnado (p.e., responsabilidad, autoestima, etc.) (Knapp, Fisher, y Levesque-Bristol, 2010; McIntyre y Sellnow, 2014), (d) el estímulo de la reflexión y el pensamiento crítico entre el alumnado (Stanton, 2014; Vaknin y Bresciani, 2013), (e) un incremento de la motivación y satisfacción del profesorado, generando cambios de actitud respecto a la enseñanza (p.e., mejora el ambiente académico y contenido educativo, aumenta el compromiso y creatividad del profesorado, etc.) (Wilczenski y Coomey, 2007), (f) una mayor conexión de la enseñanza del aula con las necesidades del entorno (McIntyre y Sellnow, 2014), y (g) el refuerzo de los lazos sociales y vínculos entre dife- 
rentes generaciones pertenecientes a una comunidad (Maloney, Myers, y Bazyk, 2014; McIntyre y Sellnow, 2014).

\section{Objetivos e hipótesis}

El objetivo de este estudio fue explorar la eficacia del PHE para mejorar las habilidades sociales y las estrategias de aprendizaje y motivación en el alumnado universitario participante. Las hipótesis establecidas fueron: (1) existirán diferencias estadísticamente significativas en las puntuaciones directas medias obtenidas por el alumnado universitario en habilidades sociales en la fase postest respecto a la fase pretest como resultado de su participación en el programa; y (2) el alumnado universitario mejorará a nivel estadísticamente significativo sus puntuaciones directas medias logradas en motivación y estrategias de aprendizaje en la fase postest respecto a la fase pretest tras participar en el PHE.

\section{Método}

\section{Participantes}

La muestra de esta investigación estuvo conformada por 78 alumnos/as universitarios/as de la Universidad de Huelva (UHU) con una media de edad de 21,05 años (rango entre 18 y 35 años). Su distribución por titulaciones fue de: (a) 27 del Grado en Educación Social (18 de segundo, 4 de tercero y 5 de cuarto curso), (b) 29 del Grado en Psicología (18 de segundo, 3 de tercero y 8 de cuarto curso), (c) 7 del Grado de Maestro en Educación Primaria (7 de tercer curso), (d) 14 del Grado en Trabajo Social (12 de segundo y 2 de tercer curso), y (e) 1 del Grado Ciencias de la Actividad Física y el Deporte (1 de tercer curso). Por sexo, 64 eran mujeres y 14 hombres.

El procedimiento para la selección de la muestra se basó en un tipo de muestreo no probabilístico denominado "muestras de conveniencia" (Martínez, 2010), seleccionando primero a la población a la que se tenía acceso, es decir, alumnado universitario perteneciente al Campus de "El Carmen" de la Universidad de Huelva (España). En segundo lugar, se seleccionaron las titulaciones relacionadas con la profesión docente que participaron en el PHE. En tercer lugar, se llevó a cabo un plan de divulgación y captación, que consistió en varias sesiones grupales en las que se informó al alumnado universitario sobre las características y condiciones de participación en el programa, solicitando paralelamente su participación voluntaria, 
lo que generó una inscripción de 102 alumnos/as universitarios/as. Y, en cuarto lugar, se seleccionaron los mentores $(N=78)$, considerando para ello los siguientes criterios: completar el acuerdo, el protocolo y las pruebas estandarizadas, demostrar interés, disponer de tiempo suficiente para desarrollar las diferentes sesiones de mentoría a lo largo del curso académico, asistir a las 3 sesiones del curso de formación y, finalmente, realizar con éxito las actividades prácticas de dicha formación.

\section{Instrumentos}

Acuerdo de participación para mentores PHE. Documento constituido por 17 cláusulas y un apartado de conformidad. Este acuerdo, tomando como referencia otros materiales similares (Fernández, 2007), fue elaborado ad hoc para el desarrollo del programa, con el propósito de establecer los derechos y deberes del alumnado universitario participante en el PHE.

Protocolo de recogida de información. Autoinforme formado por 23 ítems, de los cuales 17 ítems eran de respuesta abierta y 6 de diferentes alternativas, agrupados en 8 áreas: (a) información demográfica, (b) información académica, (c) capacitación para participar en el programa de mentoría, (d) motivo de solicitud, (e) justificación de su participación, (f) nivel de compromiso, (g) disponibilidad horaria, y (h) 1 cláusula donde se indicaba que la información aportada era veraz. Este protocolo, elaborado ad hoc para el desarrollo del programa a partir de otros materiales análogos (Fernández, 2007), se utilizó para recoger la información demográfica, académica y de interés del alumnado universitario participante.

Escala de Habilidades Sociales. Instrumento compuesto por 33 ítems agrupados en 6 escalas: (a) autoexpresión en situaciones sociales (ASS), (b) defensa de los propios derechos como consumidor (DDC), (c) expresión de enfado o disconformidad (EE/D), (d) decir no y cortar interacciones (DNCI), (e) hacer peticiones (HP), y (f) iniciar interacciones positivas con el sexo opuesto (IIPSO). Escala elaborada por Gismero (2000), que se empleó para evaluar las habilidades sociales del alumnado universitario, por su alta fiabilidad (modelo alfa: 0,89 puntos) y aceptable validez (análisis factorial confimatorio que arroja índices de ajuste adecuados sobre el modelo estructural y medidas de validez concurrente sobre autodescripciones y adjetivos). 
que conforman 15 subescalas: (a) orientación intrínseca (OI), (b) orientación extrínseca (OE), (c) valor de la tarea (VT), (d) control sobre las creencias (CC), (e) autoeficacia (Af), (f) ansiedad (A), (g) repetición y ensayo (RyE), (h) elaboración (E), (i) organización (O), (j) pensamiento crítico (PC), (k) autorregulación metacognitiva (AM), (l) tiempo y ambiente de estudio (TAE), (m) regulación del esfuerzo (RE), (n) solicitud de ayuda (SA), y (ñ) aprendizaje por pares (AP). Fue elaborado por Pintrich, Smith, García, y Mckeachie (1991) y se utilizó para evaluar la motivación y estrategias de aprendizaje del alumnado universitario, pues presenta una fiabilidad (modelo alfa - Cronbach-: entre 0,52 y 0,93 puntos en las diferentes escalas) y validez aceptable (índices de ajuste adecuados del modelo factorial confirmatorio y evidencias de validez predictiva sobre rendimiento académico).

Cuaderno de trabajo de mentores PHE. Material en el que se presentaba de forma estructurada las sesiones de mentoría: objetivos, tareas realizadas, materiales empleados, dificultades encontradas, tareas pendientes para la próxima sesión, evaluación y observaciones. Fue elaborado ad hoc para el desarrollo del programa a partir de otros materiales (Fernández, 2007; Herrera et al., 2000; Permaul, 2009) y se empleó durante las sesiones de mentoría y el plan de seguimiento del programa.

Protocolos de seguimiento 1, 2 y 3. Instrumentos compuestos por 13 ítems de respuesta cerrada y 3 de respuesta abierta, en el caso del protocolo de la sesión de seguimiento 1, y por 21 ítems de respuesta abierta, en el caso de los protocolos de las sesiones de seguimiento 2 y 3 , que se agruparon en 3 áreas que incluían los datos de identificación, valoraciónpercepción de sesiones y observaciones. Estos protocolos, que se elaboraron ad hoc para el desarrollo del plan de seguimiento del programa, tomando como referencia otros protocolos similares (Fernández, 2007), se emplearon para identificar las desviaciones que se produjeran en las sesiones de mentoría.

Hoja de grupos de discusión. Instrumento compuesto por 2 ítems de respuesta abierta que se utilizó para recoger las percepciones del alumnado universitario en el seguimiento 4 sobre las dificultades encontrados en el desarrollo de las sesiones de mentoría y las posibles soluciones. Este instrumento fue elaborado ad hoc para el desarrollo de este tipo de programas por Fernández (2007). 


\section{Procedimiento}

Este trabajo de investigación se llevó a cabo en cuatro fases (Anexo 1): análisis de necesidades, diseño y planificación del programa, aplicación del programa (procedimiento de selección de la muestra, plan de intervención, seguimiento y evaluación de resultados), y evaluación del programa (Arco y Fernández, 2007; Fernández y Arco, 2011).

El plan de intervención se compuso de dos actividades, la primera, el curso de formación de mentores, desarrollada con el alumnado universitario inscrito en el PHE y seleccionado para cursar la formación. Y, la segunda, las sesiones de mentoría, que se llevaron a cabo después de (MENTOR/National Mentoring Partnership, 2005): (a) seleccionar la muestra de mentores (cuarta etapa del muestreo), (b) establecer las parejas de mentores $(N=39)$, bajo determinados criterios (p.e., preferencias, horario académico, titulación y experiencia previa), (c) seleccionar al alumnado de educación obligatoria o "mentees", inscritos por sus docentestutores, con el consentimiento de las familias, en el segundo trimestre del curso escolar, y (d) realizar la asignación de los "mentees" a los mentores, bajo ciertos criterios (p.e., disponibilidad horaria y equivalencia en experiencia de vida e intereses de mentores-“mentees").

Respecto al curso de formación de mentores, primera actividad del plan de intervención, su propósito fue entrenar al alumnado universitario participante en las tareas que iban a desempeñar en el desarrollo de las sesiones de mentoría (Gopee, 2011). Para ello, se optó por la formación en aquellos contenidos que contribuían al éxito de este tipo de programas (Jekielek, Moore, Hair, y Scarupa, 2002; MENTOR/National Mentoring Partnership, 2005). Esta actividad constó de 3 sesiones de 3 horas de duración distribuidas en una semana, en las que se trabajaron, a través de enseñanza expositiva, análisis de casos y modelado, los siguientes contenidos: (a) sesión 1: plan de formación del curso, justificación del programa y uso del Cuaderno de trabajo de mentores PHE, (b) sesión 2: desarrollo del guión de llamada telefónica para concertar la primera sesión de mentoría entre mentores y alumnado de educación obligatoria participante y del primer encuentro con los "mentees" y sus familias, y diseño, planificación y aplicación de la evaluación de necesidades de los "mentees", y (c) sesión 3: contenidos y actividades de aprendizaje para las actividades extraescolares de carácter cocurricular con los "mentees", en función de las necesidades identificadas, es decir, refuerzo escolar en áreas instrumentales, intervención en dificultades de aprendizaje, entrenamiento en estrategias de aprendizaje, aplicación y evaluación de actividades de orientación personal, de orientación vocacional-profesional, y de gestión del ocio y tiempo libre. 
Por otro lado, las sesiones de mentoría entre cada pareja de mentores y su correspondiente "mentee", segunda actividad del plan de intervención, se iniciaron en enero y se prolongaron hasta junio del curso escolar en horario extraescolar, con una frecuencia semanal de 90 minutos de duración, en los horarios y lugares fijados desde el programa. Se realizaron un total de 481 sesiones, con una media de 12,33 sesiones (rango: 5-17 sesiones) por pareja de mentores-"mentee" ( $D T=2,98)$, de las cuales 423 fueron de carácter individual (parejas de mentores con su respectivo "mentee"), con una media de 10,84 sesiones $(D T=2,50)$ y un rango de entre 5 y 14 sesiones, y 58 grupales (varias parejas de mentores con sus respectivos "mentees" en actividades comunes, como por ejemplo, de ocio) con una media de 1,48 sesiones $(D T=0,88)$ y un rango de entre 1 y 3 sesiones. No obstante, es necesario mencionar que estas diferencias en el número de sesiones implementadas por pareja de mentores-“mentee" se deben a los diferentes momentos en los que los "mentees" fueron inscritos en el programa, así como a las sesiones que fueron canceladas $(N=77)$, de las cuales 58 fueron individuales $(M=$ $1,48 ; D T=0,94$; rango entre $1-3$ sesiones $)$ y 18 grupales $(M=0,46 ; D T=0,55$; rango entre 1 2 sesiones).

Los contenidos trabajados por los mentores en las sesiones de mentoría (Cuaderno de trabajo de mentores PHE) se adecuaron a las necesidades detectadas de los "mentees", las cuales fueron identificadas a partir de la información aportada por familias y docentes-tutores, y de la información recogida por los mentores en la primera sesión de mentoría, si bien el nivel de coincidencia con los contenidos recomendados por la literatura especializada es elevado, destacando (Boland, 2010): rendimiento escolar (p.e., refuerzo escolar, estrategias de aprendizaje cognitivas y metacognitivas, ansiedad ante los exámenes, expresión oral y escrita. etc.), orientación personal (p.e., gestión de emociones, habilidades sociales, etc.), orientación vocacional-profesional (p.e., transición de etapa educativa, itinerarios formativos, visitas a Institutos de Educación Secundaria y Universidad para conocer las características de los centros, información sobre titulaciones, etc.), y gestión del ocio y tiempo libre (p.e., actividades grupales para una mejor socialización del alumnado en su grupo-clase, resolución de conflictos, conocer diferentes alternativas de ocio, etc.).

Paralelamente al desarrollo de las sesiones de mentoría, se implementó un plan de seguimiento con el propósito de vigilar las posibles desviaciones que se produjeran en la ejecución de las mismas (Arco y Fernández, 2007). Este plan consistió en 4 medidas de seguimien- 
to obligatorio, implementadas por los responsables del programa con los mentores: (a) seguimientos 1, 2 y 3: una sesión grupal de 2 horas de duración una vez efectuada la segunda (protocolo de seguimiento 1), séptima (protocolo de seguimiento 2) y duodécima (protocolo de seguimiento 3) sesión, respectivamente, donde se valoraron las actuaciones llevadas a cabo a partir de los cuadernos y contribuciones verbales de los mentores, además de aportarles recomendaciones oportunas para el desarrollo de las siguientes sesiones de mentoría, y (b) seguimiento 4: una sesión grupal de 2 horas de duración después de finalizar las sesiones de mentoría, en la que los mentores valoraron el desarrollo global en las mismas, y se recogieron sus percepciones, tales como, dificultades y mejoras (hoja de grupos de discusión).

Por su parte, con el Plan de evaluación de resultados se tomaron medidas de las variables dependientes antes y después de la aplicación del programa para conocer el impacto del mismo (Arco y Fernández, 2007).

\section{Análisis Estadístico}

El diseño metodológico adoptado en esta investigación fue un diseño cuasiexperimental pretest-postest (Ato, 2010). Los datos fueron analizados a través del "Statistical Package for the Social Sciences", versión 20.0, realizándose la prueba $t$ para muestras relacionadas y la prueba de los rangos con signo de Wilcoxon, una vez comprobada la normalidad de la distribución de las puntuaciones a través de la prueba Kolmogorov-Smirnov.

\section{Resultados}

Los resultados de las comparaciones pretest-postest sobre habilidades sociales, hipótesis 1 , revelan diferencias estadísticamente significativas a favor de la fase postest entre las puntuaciones directas medias obtenidas en la mayoría de las escalas y la puntuación directa global de la EHS (Gismero, 2000), si bien en la escala DNCI dichas diferencias son a favor de la fase pretest $(M=15,63 ; D T=3,74)$ frente a la fase postest $(M=12,29 ; D T=2,20)$ (Tablas 1 y 2).

Tabla 1. Comparaciones Pretest-Postest sobre Habilidades Sociales (Prueba t)

\begin{tabular}{lcccccc}
\hline \multicolumn{2}{c}{ Escala/Fase } & $N$ & Media & D. Típica & $t$ & $\begin{array}{c}\text { Sig. (bila- } \\
\text { teral) }\end{array}$ \\
\hline Escala ASS & Pretest & 78 & 23,55 & 4,26 & 47,25 & $0,00 * *$
\end{tabular}




\begin{tabular}{lllllll} 
& Postest & 78 & 25,34 & 3,33 & & \\
Escala EE/D & Pretest & 78 & 10,44 & 2,57 & 36,04 & $0,00 * * *$ \\
& Postest & 78 & 14,62 & 2,55 & & \\
Escala DNCI & Pretest & 78 & 15,63 & 3,74 & 37,15 & $0,00 * *$ \\
& Postest & 78 & 12,29 & 2,20 & & \\
Escala HP & Pretest & 78 & 12,48 & 3,16 & 34,63 & $0,00 * * *$ \\
& Postest & 78 & 17,73 & 3,39 & & \\
Escala IIPSO & Pretest & 78 & 11,76 & 3,20 & 32,78 & $0,00 * *$ \\
& Postest & 78 & 14,64 & 2,76 & & \\
Escala DDC & Pretest & 78 & 8,33 & 1,54 & $-7,68$ & $0,00 * * *$ \\
& Postest & 78 & 13,92 & 2,75 & & \\
P. Directa Glo- & Pretest & 78 & 82,19 & 18,47 & 53,31 & $0,00 * *$ \\
bal & Postest & 78 & 98,24 & 11,85 & & \\
\hline Nota: $* p<0,05 * * * p<0,01 ; * * * p<0,001$ & & & & \\
& & & & &
\end{tabular}

Tabla 2. Comparaciones Pretest-Postest sobre Habilidades Sociales (Prueba z)

\begin{tabular}{lcccccc}
\hline \multicolumn{2}{c}{ Escala/Fase } & $N$ & Media & D. Típica & $z$ & $\begin{array}{c}\text { Sig. (bila- } \\
\text { teral) }\end{array}$ \\
\hline Escala DDC & Postest & 78 & 14,64 & 2,76 & $-7,68$ & $0,00 * * *$ \\
& Pretest & 78 & 8,33 & 1,54 & &
\end{tabular}

Por su parte, las comparaciones intragrupo sobre motivación y estrategias de aprendizaje, hipótesis 2, arrojan igualmente diferencias estadísticamente significativas a favor de la fase postest en gran parte de las subescalas del MSLQ (Pintrich et al., 1991), aunque en las subescalas A y RE estas diferencias son a favor de la fase pretest $(M=4,46 ; D T=1,38$ y $\mathrm{M}=$ 4,$49 ; D T=0,64$ respectivamente) frente a la fase postest $(M=3,80 ; D T=1,40$ y $M=3,88$; $D T=0,61$, respectivamente) (Tabla 3$)$.

Tabla 3. Comparaciones Pretest-Postest sobre Motivación y Estrategias de Aprendizaje

\begin{tabular}{cllcccc}
\hline \multicolumn{2}{c}{ Subescala $/$ Fase } & $N$ & Media & D. típica & $z$ & $\begin{array}{c}\text { Sig. }(2- \\
\text { tailed })\end{array}$ \\
\hline Motivación & & & & & & \\
Escala OI & Pretest & 78 & 2,93 & 0,95 & $-6,68$ & $0,00^{* *}$ \\
& Postest & 78 & 5,06 & 1,06 & & \\
Escala OE & Pretest & 78 & 3,30 & 1,24 & $-5,99$ & $0,00^{* *}$ \\
& Postest & 78 & 4,80 & 1,20 & & \\
Escala VT & Pretest & 78 & 2,55 & 0,80 & $-18,68$ & $0,00^{* * *}$ \\
& Postest & 78 & 5,57 & 0,77 & & \\
Escala CC & Pretest & 78 & 3,04 & 0,94 & $-10,16$ & $0,00^{* * *}$ \\
& Postest & 78 & 5,04 & 0,94 & $-7,44$ & $0,00^{* * *}$
\end{tabular}




\begin{tabular}{|c|c|c|c|c|c|c|}
\hline \multirow{3}{*}{ Escala A } & Postest & 78 & 5,48 & 0,78 & \multirow{3}{*}{2,20} & \multirow{3}{*}{$0,03 *$} \\
\hline & Pretest & 78 & 4,46 & 1,38 & & \\
\hline & Postest & 78 & 3,80 & 1,40 & & \\
\hline \multicolumn{7}{|c|}{ Estrategias de aprendizaje } \\
\hline \multirow[t]{2}{*}{ Escala PC } & Pretest & 78 & 3,92 & 1,18 & \multirow{2}{*}{$-2,26$} & \multirow{2}{*}{$0,03 *$} \\
\hline & Postest & 78 & 4,42 & 0,95 & & \\
\hline \multirow[t]{2}{*}{ Escala AM } & Pretest & 78 & 3,41 & 0,72 & \multirow{2}{*}{$-11,65$} & \multirow{2}{*}{$0,00 * * *$} \\
\hline & Postest & 78 & 4,91 & 0,60 & & \\
\hline \multirow[t]{2}{*}{ Escala TAE } & Pretest & 78 & 3,53 & 0,61 & \multirow{2}{*}{$-9,60$} & \multirow{2}{*}{$0,00 * * *$} \\
\hline & Postest & 78 & 4,64 & 0,57 & & \\
\hline \multirow[t]{2}{*}{ Escala RyE } & Pretest & 78 & 3,42 & 1,24 & \multirow{2}{*}{$-5,41$} & \multirow{2}{*}{$0,00 * *$} \\
\hline & Postest & 78 & 4,68 & 1,01 & & \\
\hline \multirow[t]{2}{*}{ Escala E } & Pretest & 78 & 2,79 & 1,00 & \multirow{2}{*}{$-13,80$} & \multirow{2}{*}{$0,00 * * *$} \\
\hline & Postest & 78 & 5,31 & 0,80 & & \\
\hline \multirow[t]{2}{*}{ Escala O } & Pretest & 78 & 2,99 & 1,10 & \multirow{2}{*}{$-9,13$} & \multirow{2}{*}{$0,00 * * *$} \\
\hline & Postest & 78 & 5,03 & 1,04 & & \\
\hline \multirow[t]{2}{*}{ Escala RE } & Pretest & 78 & 4,49 & 0,64 & \multirow{2}{*}{4,24} & \multirow{2}{*}{$0,00 * *$} \\
\hline & Postest & 78 & 3,88 & 0,61 & & \\
\hline \multirow[t]{2}{*}{ Escala AP } & Pretest & 78 & 3,16 & 1,19 & \multirow{2}{*}{$-10,86$} & \multirow{2}{*}{$0,00 * * *$} \\
\hline & Postest & 78 & 5,19 & 1,00 & & \\
\hline
\end{tabular}

\section{Discusión y conclusiones}

Esta experiencia educativa surgió con el propósito de ofrecer a los estudiantes universitarios acceso a un tipo de experiencias de aprendizaje facilitadores de la adquisición y dominio de dos grupos de habilidades y competencias claves relacionadas con la profesión docente, como son las habilidades sociales y de aprender a aprender. En esta línea, a la luz de los resultados obtenidos, se pueden establecer las siguientes conclusiones. Primero, las diferencias estadísticamente significativas entre las puntuaciones directas medias obtenidas por el alumnado universitario participante en la fase postest respecto a la fase pretest en la mayor parte de las escalas de la EHS (Gismero, 2000), permiten aceptar la hipótesis uno parcialmente. Segundo, respecto a la hipótesis 2 , el carácter significativo de las diferencias encontradas en la fase postest respecto a la fase pretest en gran parte de las subescalas del MSLQ (Pintinch et al., 1991), permite igualmente aceptar parcialmente esta hipótesis.

Estos resultados coinciden en gran medida con los que se deriban de la literatura especializada cuando se emplea el A-S y la mentoría entre iguales como base de un programa de intervención (McIntyre y Sellnow, 2014; Weiler, Zarich, Haddock, Krafchick, y Zimmerman, 2014; Westover, 2012). Por ejemplo, el fomento de aspectos relacionados con el desarrollo 
personal y social del alumnado como el aumento en la responsabilidad, mejora de la autoestima, así como una mayor conexión de la enseñanza del aula con las necesidades del entorno, además de proveer oportunidades significativas con la comunidad en las que se enseñan responsabilidades cívicas y formentan la participación ciudadana durante toda la vida, y donde se proporcionan oportunidades de aprendizaje significativo de la vida real, la práctica de habilidades importantes y una vital compresión social para los estudiantes, también el incremento de compentencias como la comunicación y habildiades de resolución de problemas, además de aumentrar sus habilidades interpersonales, el éxito académico, el desarrollo profesional y respuestas emocionales adecuadas.

Los resultados logrados se alinean con los planteados por Betina y Contini (2011), que señalan la importancia del contexto en sus múltiples acepciones como determinante de cómo se aprende la habilidad y de cuánto se practica la habilidad hasta convertirse en competencia. Por tanto, al igual que plantean García (2010), Gomes y Soares (2013), Ordaz (2013), y Soares, Francischetto, Peçanha, Miranda, y Dutra (2013), el hecho de practicar en ámbitos académicos la conducta asertiva repercute positivamente en las habilidades sociales.

En este sentido, la participación en el PHE ha implicado para el alumnado universitario un cambio y adaptación constante a las dificultades que se han presentado durante el desarrollo del mismo, resolución de conflictos, toma de decisiones, mayor compromiso con su trabajo, etc., que están a la base de las mejoras que se han generando en sus habilidades sociales. Sin embargo, los resultados obtenidos, comparados con otros trabajos con objetivos similares (p.e., Gaeta y López, 2013), revelan un nivel bajo en habilidades de autoexpresión en situaciones sociales y hacer peticiones, lo que implica que no se ha producido un avance ostensible en el alumnado participante a la hora de reconocer las emociones en los otros, produciéndose asimismo un deterioro en habilidades de decir no y cortar interacciones. Estos resultados se pueden explicar como consecuencia de una percepción demasiado elevada de sí mismos sobre sus habilidades sociales en la fase pretest, antes de someterse a situaciones reales de la práctica educativa, lo que ha podido generar un reajuste a la baja en sus creencias respecto al dominio de algunas de sus habilidades sociales.

Por su parte, las estrategias de aprendizaje y motivación (p.e., pensamiento crítico, regulación del esfuerzo, autorregulación metacognitiva, etc.) se asocian, no solo a la competencia de aprender a aprender, sino también a otras competencias clave para el aprendizaje per- 
manente (p.e., sentido de la iniciativa y espíritu de empresa) (Comunidades Europeas, 2007), por lo que los resultados derivados de la participación en el PHE, además de generar mejoras en la competencia aprender a aprender del alumnado universitario, es posible que hayan estimulado el desarrollo de otras competencias clave (p.e., competencias digital). Igualmente, las mejoras conseguidas en la motivación intrínseca (p.e., metas de orientación intrínseca, valor de la tarea y control de las creencias de aprendizaje) de los participantes, y su fuerte relación con el uso de estrategias cognitivas y de autorregulación, han generado una mayor motivación para aprender, tal y como han mostrado otros estudios previos (Pintrich y De Groot, 1990). No obstante, también se debe considerar el deterioro que se ha producido en regulación del esfuerzo, que de nuevo puede explicarse por una elevada percepción del nivel competencial de los mentores en la fase pretest, generándose un reajuste en las creencias después de participar en las situaciones reales de la práctica profesional docente planteadas desde el PHE.

Para una interpretación más precisa de los resultados obtenidos es necesario hacer alusión a la posible presencia de algunos factores ligados al diseño metodológico empleado que pueden debilitar la validez interna de los datos (p.e., historia, maduración, administración de pruebas, etc.), entre los que se pueden destacar: (a) los diferentes motivos del alumnado universitario para participar en el programa, (b) el tiempo de participación en el programa, e incluso en mayor medida las diferencias en el número de sesiones de mentoría llevadas a cabo con el alumnado de educación obligatoria, (c) la participación de los mentores en diferentes tipos de sesiones de mentoría, de carácter individual y grupal, y (d) la diversidad de las experiencias de aprendizaje de los mentores, determinadas por las necesidades de los "mentees" y los contenidos abordados en las sesiones de mentoría.

Por tanto, para próximas ediciones del PHE, con el propósito de aumentar la validez interna y, por ende, la potencia de los resultados, sería conveniente seleccionar diseños metodológicos con mayor grado de experimentalidad (p.e., diseño cuasiexperimental pretestpostest con grupo control no equivalente) y realizar cambios que permitan a los mentores un mayor tiempo de participación, así como un número similar de sesiones de mentoría (p.e., comenzar a implementar las sesiones de mentoría al inicio del curso escolar con todos los participantes, lo que implicaría cambios en la temporización del programa, sobre todo en el procedimiento de selección de la muestra, donde sería necesario mejorar la eficiencia del proceso de divulgación y captación), con una participación igualitaria en los diferentes tipos de sesiones de mentoría. 
En definitiva, esta experiencia puede considerarse, a pesar de las limitaciones mencionadas, un ejemplo de buenas prácticas sobre la compleja y demandante tarea de cómo introducir en Educación Superior metodologías de enseñanza que faciliten la transferencia de los conocimientos adquiridos por el alumnado en el ámbito formal al contexto real y a las necesidades de la comunidad, estimulando al mismo tiempo la adquisición y mejora de las competencias previstas en los planes de estudio de las titulaciones (Miñaca et al., 2011). De esta forma, en los mismos términos que plantean Blázquez, Chamizo, Cano, y Gutiérrez (2013), estaremos contribuyendo de manera eficiente y clara al desarrollo de la llamada tercera misión de la Universidad. Especialmente, en su dimensión de facilitar que el alumnado desarrolle las competencias adecuadas a su perfil profesional, para así mejorar su perfil de empleabilidad y, por consiguiente, satisfacer la demanda del mercado laboral.

\section{Referencias}

Arco, J. L., y Fernández, F. D. (2007). Evaluación de programas en educación no formal. En C. Torres, y J. A. Pareja (Coords.), La educación no formal y diferenciada. Fundamentos didácticos y organizativos (pp. 667-690). Madrid: Editorial CCS.

Ato, M. (2010). Tipología de diseños cuasiexperimentales. En M. T. Anguera, J. Arnau, M. Ato, T. Martínez, J. Pascual, y G. Vallejo (Eds.), Métodos de investigación en Psicología (pp. 245-269). Madrid: Síntesis.

Betina, A., y Contini, N. (2011). Las habilidades sociales en niños y adolescentes. Su importancia en la prevención de trastornos psicopatológicos. Fundamentos en Humanidades, 12(23), 159-182. Recuperado de http://www.redalyc.org/pdf/184/18424417009.pdf

Blázquez, J. J., Chamizo, J., Cano, E. I., y Gutiérrez, S. (2013). Calidad de vida universitaria: Identificación de los principales indicadores de satisfacción estudiantil. Revista de Educación, 362, 458-484. doi:10.4438/1988-592X-RE-2013-362-238.

Boland, J. (2010). Teaching and learning through civic engagement: Prospects for sustainability in teacher education. Issues in Educational Research, 20(1), 1-20. Recuperado de http://www.iier.org.au/iier20/boland.pdf

Comunidades Europeas. (2007). Competencias clave para el aprendizaje permanente. Un marco de referencia europeo. Luxemburgo: Oficina de Publicaciones Oficiales de las Comunidades Europeas. Recuperado de http://eur-lex.europa.eu/legalcontent/ES/TXT/?uri=URISERV\%3Ac11090 
Consejo Económico y Social de España. (2014). Memoria sobre la situación socioeconómica $y$ laboral. Madrid: Consejo Económico y Social. Recuperado de http://www.ces.es/documents/10180/2772085/Memoria_Socioeconomica_CES2014.p df

European Commission. (2013). European social statistics. Luxembourg: Publications Office of the European Union. Recuperado de http://ec.europa.eu/eurostat/documents/3930297/5968986/KS-FP-13-001-EN.PDF/

Fernández, F. D. (2007). La tutoría entre compañeros en la Universidad (Tesis doctoral, Universidad de Granada). Recuperado de http://hera.ugr.es/tesisugr/16613375.pdf

Fernández, F. D., y Arco, J. L. (2011). Efectos de un programa de acción tutorial entre universitarios. Infancia y Aprendizaje, 34(1), 109-122. doi:10.1174/021037011794390120

Freire, M. J., Teijeiro, M. M., y País, C. (2013). La adecuación entre las competencias adquiridas por los graduados y las requeridas por los empresarios. Revista de Educación, 362, 13-41. doi:10.4438/1988-592X-RE-2011-362-151

Gaeta, M. L., y López, C. (2013). Competencias emocionales y rendimiento académico en estudiantes universitarios. Revista Electrónica Interuniversitaria de Formación del Profesorado, 16(2), 13-25. doi:10.6018/reifop.16.2.181031

García, A. D. (2010). Estudio sobre la asertividad y las habilidades sociales en el alumnado de Educación Social. XXI, Revista de Educación, 12, 225-240.

Gismero, E. (2000). Escala de habilidades sociales. Madrid: TEA.

Gomes, G., y Soares, A. B. (2013). Intelligence, social skills and academic expectations in university students' performance. Psicologia-Reflexao e Critica, 26(4), 780-789. doi:10.1590/S0102-79722013000400019

Gopee, N. (2011). Mentoring and supervision in healthcare. London: SAGE Publications.

Herrera, C., Sipe, C. L., y McClanahan, W. S. (2000). Mentoring and relationship building. Alexandria, VA: MENTOR/National Mentoring Partnership.

Instituto Nacional de Estadística. (2015). Encuesta de condiciones de vida (ECB-2014). Resultados definitivos. Recuperado de http://www.ine.es/prensa/np908.pdf

Jekielek, S. M., Moore, K. A., Hair, E. C., y Scarupa, H. J. (2002). Mentoring: A promising strategy for youth development [research brief]. Washington, DC: Child Trends.

Jurado, P., Olmos, P., y Pérez, A. (2014). Los jóvenes en situación de vulnerabilidad y los programas formativos de transición al mundo del trabajo. Educar 2015, 51(1), 211224. doi:10.5565/rev/educar.648

Knapp, T., Fisher, B., y Levesque-Bristol, C. (2010). Service-learning's impact on college 
students' commitment to future civic engagement, self-efficacy, and social empowerment. Journal of Community Practice, 18, 233-251. doi:10.1080/10705422.2010.490152

Maloney, S. M., y Griffith, K. (2013). Occupational therapy students' development of therapeutic communication skills during a service-learning experience. Occupational Therapy in Mental Health, 29, 10-26. doi:10.1080/0164212X.2013.760288

Maloney, S. M., Myers, C., y Bazyk, J. (2014). The influence of a community-based servicelerning experience on the development of occupational therapy students' feelings of civic responsibility. Occupational Therapy in Mental Health, 30(2), 144-161. doi:10.1080/0164212X.2014.910160

Martínez, R. (2010). El método de encuestas por muestreo: Conceptos básicos. En M. T. Anguera, J. Arnau, M. Ato, R. Martínez, J. Pascual, y G. Vallejo (Eds.), Métodos de investigación en Psicología (pp. 385-431). Madrid: Síntesis.

McIntyre, K. A., y Sellnow, D. D. (2014). A little bit can go a long way: An examination of required service in the basic communication course. Communication Teacher, 28(1), 57-73. doi:10.1080/17404622.2013.843012

MENTOR/National Mentoring Partnership. (2005). Cómo construir un programa de mentoría exitoso utilizando los elementos de la práctica efectiva. Alexandria, VA: MENTOR/National Mentoring Partnership. Recuperado de http://www.mentoring.org/newsite/wp-content/uploads/2015/09/Full_Toolkit_SPA.pdf

Ministerio de Educación, Cultura y Deporte. (2014). Panorama de la Educación. Indicadores de la OCDE 2014. Madrid: Secretaría General Técnica. Subdirección General de Documentación y Publicaciones, Ministerio de Educación, Cultura y Deporte. Recuperado de http://www.mecd.gob.es/dctm/inee/indicadoreseducativos/panorama2014/panorama2014web.pdf?documentId=0901e72b81b20622

Ministerio de Educación, Cultura y Deporte. (2015). Datos básicos del sistema universitario Español. Curso 2014-2015. Madrid: Secretaría General Técnica, Ministerio de Educación, Cultura $\quad$ D $\quad$ Deporte. Recuperado de http://www.mecd.gob.es/dms/mecd/educacion-mecd/areaseducacion/universidades/estadisticas-informes/datos-cifras/Datos-y-Cifras-del-SUECurso-2014-2015.pdf

Miñaca, M., Hervás, M., Ferrer, D., Vílchez, F., Aparicio, C. Arco, J. L., y Fernández, F. D. (2011, Mayo). El Aprendizaje-Servicio como oportunidad de aprendizaje y adquisición de competencias para el alumnado universitario. Comunicación presentada al I 
Congreso Internacional sobre Voluntariado Socio-Educativo, Granada.

Mourshed, M., Patel, J., y Suder, K. (2014). Education to employment: Getting Europe's youth into work. New York, NY: Mckinsey Center for Goverment.

Ordaz, M. (2013). La educación de habilidades sociales desde la extensión universitaria: Propuesta de acciones. Educarem Revista, 50, 269-283. Recuperado de http://ojs.c3sl.ufpr.br/ojs2/index.php/educar/article/view/32691

Permaul, J. S. (2009). Theoretical bases for service-learning: Implications for program design and effectiveness. New Horizons in Education, 57(3), 1-7. Recuperado de http://files.eric.ed.gov/fulltext/EJ893699.pdf

Pintrich, P., Smith, D., García, T., y McKeachie, W. (1991). A manual for the use of the Motivated Strategies for Learning Questionnaire (MSLQ). National Center for Research to Improve Postsecondary Teaching and Learning. University of Michigan.

Pintrich, P. R., y De Groot, E. V. (1990). Motivational and self-regulated learning components of classroom academic performance. Journal of Educational Psychology, 82(1), 33-40. doi:10.1037/0022-0663.82.1.33

Sandford, S., Copps, J., y Yeowart, C. (2007). Lean on me. Mentoring for young people at risk. A guide for donors and funders. London: New Philantropy Capital. Recuperado de

http://scottishmentoringnetwork.co.uk/assets/downloads/resources/LeanOnMe_NewPh ilanthropyCapital_Inclusion_Youth_2007.pdf

Soares, A. B., Francischetto, V., Peçanha, A. P. C. L., Miranda, J. M., y Dutra, B. M. S. (2013). Intelligence and social competence in university adaptation. Estudios de Psicología, 30(3), 317-328. doi:10.1590/S0103-166X2013000300001

Stanton, T. K. (2014). Practice makes perfect: Classrooms and curricula that generate civic minds and actions. The Journal of General Education, 63(1), 35-46. doi:10.1353/jge.2014.0001

Vaknin, L. W., y Bresciani, M. J. (2013). Implementing quality service-learning programs in Community Colleges. Community College Journal of Research and Practica, 37(12), 979-989. doi:10.1080/10668926.2010.515515

Weiler, L. M., Zarich, K. J., Haddock, S. A., Krafchick, J. L., y Zimmerman, T. S. (2014). A comprehensive model of mentor experiences: Perceptions, strategies, and outcomes. Journal of Community Psychology, 42(5), 593-608. doi:10.1002/jcop.21640.

Westover, J. H. (2012). Academic service-learning across disciplines. Models, outcomes and assessment. Champaign, IL: Common Groum Publishing. 
Wilczenski, F. L., y Coomey, S. M. (2007). A practical guide to service learning: Strategies for positive development in schools. New York, NY: Springer.

Zimmerman, B. J. (2002). Becoming self-regulated learned: An overview. Theory into Practice, 41, 64-72. doi:10.1207/s15430421tip4102_2 
Anexo 1

\begin{tabular}{|c|c|c|}
\hline \multicolumn{3}{|c|}{ Estructura del PHE } \\
\hline & Fase & Actuaciones \\
\hline \multicolumn{2}{|c|}{$\begin{array}{l}\text { Análisis de necesidades y } \\
\text { conclusión diagnóstica }\end{array}$} & $\begin{array}{l}\text { - Definición del contexto en el que se integraría el programa } \\
\text { - Identificación de la población diana y análisis de recursos } \\
\text { - Detección de necesidades, estableciendo el tipo de necesidades } \\
\text { y sus dimensiones } \\
\text { - Establecimiento de prioridades y emisión de una conclusión } \\
\text { diagnóstica }\end{array}$ \\
\hline \multicolumn{2}{|c|}{ Metas y objetivos } & $\begin{array}{l}\text { - Establecimiento de metas y objetivos que respondían a las ne- } \\
\text { cesidades detectadas }\end{array}$ \\
\hline \multicolumn{2}{|c|}{$\begin{array}{l}\text { Diseño y planificación del } \\
\text { programa }\end{array}$} & $\begin{array}{l}\text { - Determinación del organigrama del personal responsable del } \\
\text { programa } \\
\text { - Elaboración del modelo de impacto: hipótesis causal, de inter- } \\
\text { vención y de acción } \\
\text { - Definición de la población diana } \\
\text { - Selección de los diseños metodológicos de intervención } \\
\text { - Procedimiento de selección de la muestra } \\
\text { - Plan de intervención } \\
\text { - Plan de seguimiento } \\
\text { - Plan de evaluación de resultados }\end{array}$ \\
\hline \multirow{4}{*}{ 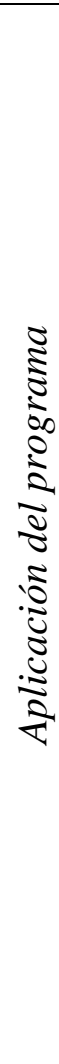 } & $\begin{array}{l}\text { Procedimiento de se- } \\
\text { lección de la muestra }\end{array}$ & $\begin{array}{l}\text { - Etapa 1: selección de unidades muestrales } \\
\text { - Etapa 2: plan de divulgación y captación } \\
\text { - Etapa 3: Selección de alumnado que accede a formación de } \\
\text { mentores } \\
\text { - Etapa 4: Selección de la muestra, establecimiento de parejas de } \\
\text { mentores y asignación de "mentees" }\end{array}$ \\
\hline & $\begin{array}{l}\text { Actividades del plan } \\
\text { intervención }\end{array}$ & $\begin{array}{l}\text { - Sesiones del curso de formación de mentores } \\
\text { - Sesiones de mentoría individuales y grupales entre parejas de } \\
\text { mentores y "mentees": rendimiento escolar, orientación perso- } \\
\text { nal, orientación vocacional-profesional, y gestión del ocio y } \\
\text { tiempo libre }\end{array}$ \\
\hline & $\begin{array}{l}\text { Medidas del plan de } \\
\text { seguimiento }\end{array}$ & $\begin{array}{l}\text { - Sesión grupal de seguimiento 1: valoración de las actuaciones } \\
\text { de las sesiones de mentoría 1-2 } \\
\text { - Sesión grupal de seguimiento 2: valoración de las actuaciones } \\
\text { de las sesiones de mentoría 3-7 } \\
\text { - Sesión grupal de seguimiento 3: valoración de las actuaciones } \\
\text { de las sesiones de mentoría 8-12 } \\
\text { - Sesión grupal de seguimiento 4: valoración del desarrollo glo- } \\
\text { bal de las sesiones de mentoría }\end{array}$ \\
\hline & $\begin{array}{l}\text { Actuaciones del plan } \\
\text { de evaluación de re- } \\
\text { sultados }\end{array}$ & $\begin{array}{l}\text { Toma de medidas de las variables dependientes antes y después } \\
\text { de la aplicación del programa a través de pruebas estandarizadas }\end{array}$ \\
\hline \multicolumn{2}{|c|}{ Evaluación proceso } & $\begin{array}{l}\text { Proporcionar información acerca del desarrollo de las activida- } \\
\text { des, y valoración del programa (deficiencias y suficiencias del } \\
\text { diseño, etc.) }\end{array}$ \\
\hline \multicolumn{2}{|c|}{ Evaluación impacto } & $\begin{array}{l}\text { Establecer el tipo, dirección y magnitud de los cambios produci- } \\
\text { dos por la aplicación del programa }\end{array}$ \\
\hline \multicolumn{2}{|c|}{ Evaluación resultados } & Comunicación de resultados \\
\hline
\end{tabular}

